\title{
A Predator-Prey Model with General Holling Interactions in Presence of Additional Food
}

\author{
Banshidhar Sahoo \\ Department of Mathematics, Daharpur A.P.K.B Vidyabhaban, Paschim Medinipur, West Bengal, India
}

\begin{abstract}
A predator-prey model with general Holling type of interactions in presence of additional food is proposed. The stability of equilibrium points of the system is analysed. The bifurcation analysis is done with respect to Holling parameter as well as quantity of additional food. The model will be useful for construction of real food chain model for predicting future which will be important for bio-conservation and pest management.
\end{abstract}

Keywords Predator-Prey, Additional Food, General Holling, Stability

\section{Introduction}

Understanding the relationship between predator and prey is a central goal in ecology. One significant component of the predator-prey relationship is the predator's feeding rate upon prey. The predator's feeding rate as a function of changes in the density of food items in the habitat is called organism's "functional response". There are many conceptual food chain models considering specific functional form of predator-prey interaction.

Many scholars have studied about continuous food chain models for several functional responses such as Holling-Tanner type[1,2], Beddington-DeAngelis type[3,4] and ratio dependent type[5,6]. But we observe that these functions satisfy some basic biological requirements and have the advantages of mathematical simplicity. However, there is no biological reason to prefer this special type of functional responses, but the exact predator functional response is more important.

A realistic interaction function should be based on biological hypotheses. Interaction function should be such that it does not allow the predator to grow arbitrarily fast, even if prey is abundant. Furthermore, the shape of the function for low prey densities can often be guessed. In the intermediate region, between very low and very high prey densities, the interaction function in most cases expected to be increasing [7]. In this sense the Holling functions are not the only realistic interaction functions, but merely the simplest of realistic approximations. Many scholars have studied a food chain model with Holling type-I, type-II or type-III functional responses. The Holling type-II function is based on the

* Corresponding author:

banshivu@gmail.com (Banshidhar Sahoo)

Published online at http://journal.sapub.org/plant

Copyright (C) 2012 Scientific \& Academic Publishing. All Rights Reserved assumption that predation rate is proportional to prey density if prey is scarce. If predator actively seeks out large concentrations of prey the Holling type-III are more appropriate. But the functional responses should be of general type[7] for constructing a real food chain model. In fact, in real world, predators of different species may feed on preys in different types of consumption ways. We have now concentrated about two species food chain model with general Holling type of functional responses. The predator-prey model with general Holling interactions is of the form:

$$
\begin{gathered}
\frac{d X}{d T}=R X\left(1-\frac{X}{K}\right)-\frac{A X^{n} Y}{B+X^{n}} \\
\frac{d Y}{d T}=\frac{C X^{n} Y}{B+X^{n}}-D Y
\end{gathered}
$$

where $n(>0)$ is Holling parameter. The variables " $\mathrm{X}$ " and "Y" are the density of prey and predator species respectively. " $T$ " is the time. The constant " $R$ " and " $K$ " are the intrinsic growth rate and carrying capacity of the prey species " $\mathrm{X}$ ". " $\mathrm{A}$ " denotes the food intake rate of the predator " $Y$ " on the prey " $\mathrm{X}$ " and " $\mathrm{C}$ " denotes the conversion rate of the prey to the predator. The constant " $\mathrm{B}$ " is the half saturation constant for the predator. " $\mathrm{D}$ " denotes the predator's death rate in absence of prey. If we consider constants ' $h_{1}$ ' and ' $e_{1}$ ' are as handling time of the predator per prey item and ability of the predator to detect the prey then we have " $\mathrm{A}$ " and "B", representing the food intake rate of predation and half saturation constant of the predator, to be $1 / h_{1}$ and $1 / h_{1} e_{1}$, respectively[8].

Now, we assume that the predator is provided with additional food of biomass " $F$ " which is assumed to be distributed uniformly in the habitat. We assume that the number of encounters per predator with the additional food is proportional to the density of the additional food. Here, the proportionality constant characterizes the ability of the predator to identify the additional food. Therefore, we have the following predator-prey model: 


$$
\begin{gathered}
\frac{d X}{d T}=R X\left(1-\frac{X}{K}\right)-\frac{A X^{n} Y}{B+\alpha \mu F+X^{n}} \\
\frac{d Y}{d T}=\frac{C\left(X^{n}+\mu F\right) Y}{B+\alpha \mu F+X^{n}}-D Y
\end{gathered}
$$

If we assume that the constants ' $h_{2}$ ' and ' $e_{2}$ ', respectively, represent the handling time of the predator per unit quantity of additional food and ability for the predator to detect the additional food, then we have $\mu=e_{2} / e_{1}$ and $\alpha=h_{2} / h_{1}$. Here the term " $\mu \mathrm{F}$ " represents effectual additional food level.

We non-dimensionlize the system (2) using

$X=B^{\frac{1}{n}} x, Y=B^{\frac{1}{n}} y$ and $t=R T$. The system (2) reduces to the following form

$$
\begin{gathered}
\frac{d x}{d t}=x\left(1-\frac{x}{\gamma}\right)-\frac{a x^{n} y}{1+\alpha \xi+x^{n}} \\
\frac{d y}{d t}=\frac{b\left(x^{n}+\xi\right) y}{1+\alpha \xi+x^{n}}-d y
\end{gathered}
$$

Where, $a=\frac{A}{R}, \gamma=\frac{K}{B^{\frac{1}{n}}}, \xi=\frac{\mu F}{\mathrm{~B}}, b=\frac{C}{R}, d=\frac{D}{R}$.

Here " $\xi$ " and " $\alpha$ " are noted as control parameters which characterize the "quantity" of additional food available to the predator and its "quality" relative to the prey respectively. The system (3) is to be analysed for $x(t) \geq 0$, $y(t) \geq 0$. In this paper, we first analyse stability criteria of the system (3) theoretically in section 2 . The numerical study is done with respect general Holling parameter and quantity of additional food. Finally conclusion is given in section 4 .

\section{Theoretical Study}

\subsection{Dissipativeness}

Obviously, the right-hand sides of the system (3) are continuous and have continuous partial derivatives on the state space $\measuredangle^{2}=\left\{(\mathrm{x}, \mathrm{y})^{\mathrm{T}}: \mathrm{x} \geq 0, \mathrm{y} \geq 0\right\}$. In fact, they are Lipschitzian on $\measuredangle^{2}+$ and then the solution of the system (3) with non-negative initial condition exists and is unique. As the solution of the system (3) initiating in the non-negative quadrant it is bounded, using [9] it is easy to see that $\measuredangle^{2}+$ is an invariant domain of the system (3).

Theorem 2.1. The system (3) is dissipative.

Proof: From the first equation of the system (3), it easy to see that $\frac{d x}{d t} \leq x\left(1-\frac{x}{\gamma}\right)$. By comparison theorem, we have $x(t) \leq \frac{\gamma}{1+c e^{-t}}$ for all $t \geq 0$, where $c=\frac{\gamma}{x_{0}}-1$. This implies that $x(t) \leq \gamma$, for sufficiently large $t$.

Considering $(x(t), y(t))$, be any solution of the system (3) with positive initial condition, we define

$$
W=\frac{1}{a} x(t)+\frac{1}{b} y(t) .
$$

That is $\frac{d W}{d t} \leq \frac{2 x}{a}-\frac{x}{a}+\left(\xi-\frac{d}{b}\right) y$.

Thus $\frac{d W}{d t}+\theta W \leq \frac{2 \gamma}{a}$, where $\theta=\min \left\{\frac{1}{a}, \frac{d}{b}-\xi\right\}$.

Applying the theory of differential inequality, we obtain

$$
0<W \leq \frac{2 \gamma}{a \theta}\left(1-e^{-\theta t}\right)+W(x(0), y(0)) .
$$

For $t \rightarrow \propto$, we have $0<W \leq \frac{2 \gamma}{a \theta}$.

Hence all solutions of the system (3) that initiated in $\measuredangle^{2}+$ are confined in the region
$S=\left\{(x, y): W=\frac{2 \gamma}{a \theta}+\eta\right.$, for all $\left.\eta>0\right\}$, which means that all species are uniformly bounded for any initial value in $\measuredangle 2+$.

\subsection{Stability Analysis}

The system (3) possesses the following steady states:

i) The trivial state $E_{0} \equiv(0,0)$. The variational matrix $V\left(E_{0}\right)$ at $E_{0}$ is given by

$$
V\left(E_{0}\right)=\left[\begin{array}{cc}
1 & 0 \\
0 & \frac{b \xi}{1+\alpha \xi}-d
\end{array}\right],
$$

which has one eigen value 1 and so $E_{0}$ is unstable.

ii) The axial state $E_{1} \equiv(\gamma, 0)$. The variational matrix $V\left(E_{1}\right)$ at $E_{1}$ is given by

$$
V\left(E_{1}\right)=\left[\begin{array}{cc}
-1 & -\frac{a \gamma^{n}}{1+\alpha \xi+\gamma^{n}} \\
0 & \frac{b\left(\gamma^{n}+\xi\right)}{1+\alpha \xi+\gamma^{n}}-d
\end{array}\right],
$$

which has one eigen value -1 and other is $\frac{b\left(\gamma^{n}+\xi\right)}{1+\alpha \xi+\gamma^{n}}-d$. The equilibrium point $E_{1}$ is said to be unstable if $\frac{b\left(\gamma^{n}+\xi\right)}{1+\alpha \xi+\gamma^{n}}>d$. i.e. $\gamma^{n}>\frac{d(1+\alpha \xi)-b \xi}{b-d}$, for $b>d$ and $1+\alpha \xi-\xi>0$, which is the existence criteria of interior equilibrium point.

iii) The interior equilibrium point $E^{*} \equiv\left(x^{*}, y^{*}\right)$,

where $x^{* n}=\frac{d(1+\alpha \xi)-b \xi}{b-d}$

and $y^{*}=\frac{\left(1+\alpha \xi+x^{* n}\right)}{a}\left(1-\frac{x^{*}}{\gamma}\right) x^{* 1-n}$. The existence criteria of $E^{*}$ reveals that $x^{*}$ exists if $b>d, 1+\alpha \xi-\xi>0$ and $y^{*}$ exists provided $x^{*} \leq \gamma$, from theorem 2.1 .

Now, we study the stability criteria around $E^{*}$. The variational matrix of $V\left(E^{*}\right)$ is given by

$$
V\left(E^{*}\right)=\left[\begin{array}{ll}
a_{11} & a_{12} \\
a_{21} & a_{22}
\end{array}\right]
$$

where $\quad a_{11}=1-\frac{2 x^{*}}{\gamma}-\frac{n a(1+\alpha \xi) x^{* n-1} y^{*}}{\left(1+\alpha \xi+x^{* n}\right)^{2}}$,

$$
\begin{gathered}
a_{12}=-\frac{a x^{* n}}{1+\alpha \xi+x^{* n},} \\
a_{21}=\frac{n b(1+\alpha \xi-\xi) x^{* n-1} y^{*}}{\left(1+\alpha \xi+x^{* n}\right)^{2}},
\end{gathered}
$$

and $\quad a_{22}=0$.

The characteristic equation of $V\left(E^{*}\right)$ is

$$
\lambda^{2}+\sigma_{1} \lambda+\sigma_{2}=0,
$$

where $\sigma_{1}=-a_{11}$ and $\sigma_{2}=-a_{12} a_{21}$.

The eigen values are $\lambda_{1,2}=\frac{-\sigma_{1} \pm \sqrt{\sigma_{1}^{2}-4 \sigma_{2}}}{2}$.

Here $a_{12}<0, a_{21}>0$ and if $a_{11}<0$, then both $\sigma_{1}>0$ and $\sigma_{2}>0$. Therefore, two eigen values are negative. Thus the system may be locally asymptotically stable around $E^{*}$ if $a_{11}<0$ otherwise it is unstable. Thus depending upon system parameters; the system may exhibit stable or unstable behaviour in this case. 


\section{Numerical Results}

In this section, we have done bifurcation analysis of the system (3) with a set of hypothetical ecosystem parameters, most of which are taken from Srinivasu et al.[8]. The parameter values are taken as $\gamma=6.0, \beta=0.3, \mathrm{~d}=0.2$, which are remain unchanged throughout simulations. We have done bifurcation analysis with respect to Holling parameter $n$ as well as quantity of additional food $\xi$.

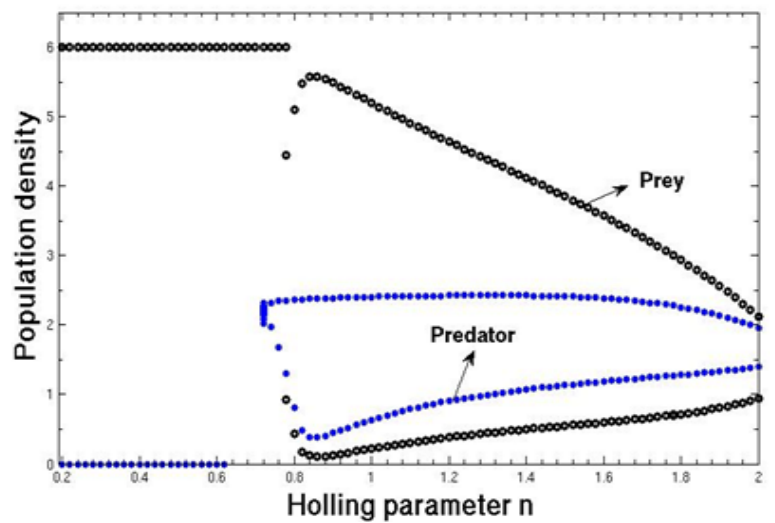

Figure 1. The bifurcation diagram of the system (3) with respect to Holling parameter $n$ without supply of additional food

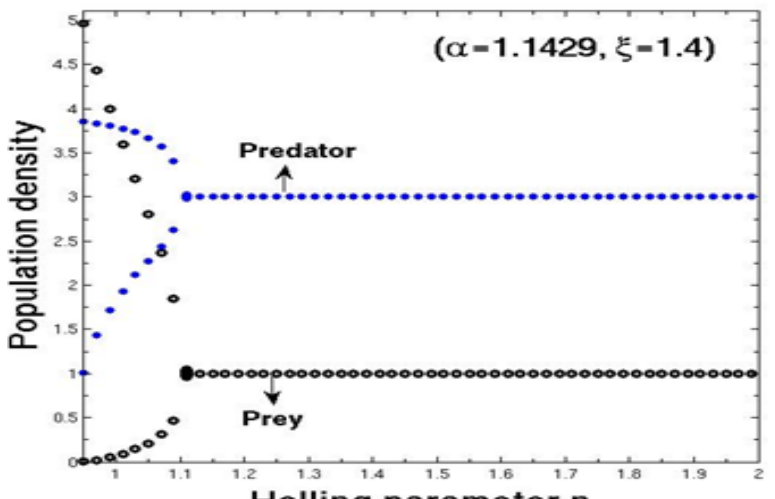

Holling parameter $\mathbf{n}$

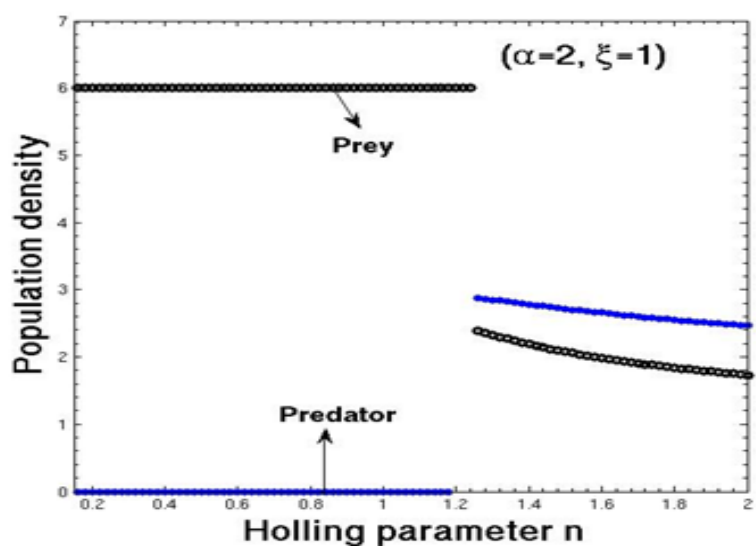

Figure 2. The bifurcation diagram of the system (3) with respect to Holling parameter $n$ in presence of additional food to predator

The figure 1 is the bifurcation diagram of the system (3) with respect to Holling parameter $n$ for $0.2 \leq n \leq 2$ without supplying any additional food. From the figure 1, we observe that the prey species has stable dynamics where the predator species extinct for $0.2 \leq n<0.61$. Within
$0.72 \leq n \leq 2$, both prey and predator species have limit cycle oscillations. On the other hand, figure 2 is the bifurcation diagram of the system (3) with respect to Holling parameter $n$ in presence of additional food to predator. The figure 2 shows that both prey and predator species have limit cycle oscillation within $0.97 \leq n \leq 1.12$ and both settles down to steady state for $1.12<n \leq 2$ in presence of additional food $\alpha=1.1429, \xi=1.4$. Again from the figure 2 , we observe that prey species have steady state while the predator species extinct from the system for $0.2 \leq n<1.19$ in presence of additional food $\alpha=2, \xi=1$ and both the species have stable dynamics for $1.19 \leq n \leq 2$. Figure 3 is the bifurcation diagram of the system (3) with respect to quantity of additional food $\xi$ to the predator for different values of $n$ and $\alpha=2$. The figure 3 indicates that the system have either limit cycle or steady state in ranges of $0 \leq \xi \leq 1$. From figure 3 , it is important to note that there are no eradication effects in the system in presence of additional food for different functional responses. Therefore, survival of the species in a system depends on interaction functions as well as supply of additional food.
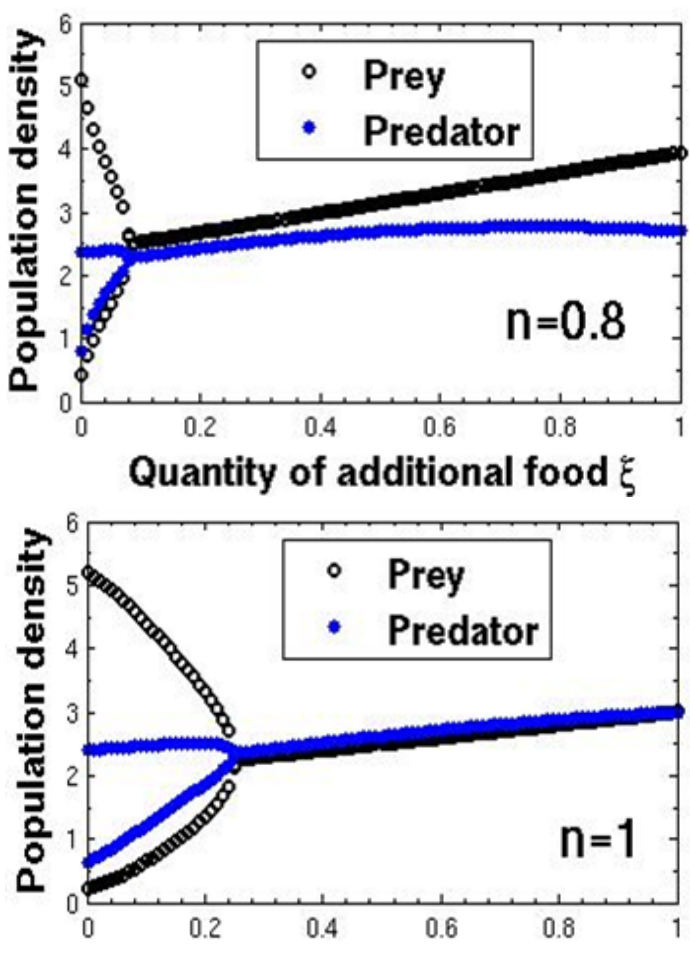

Quantity of additional food $\xi$

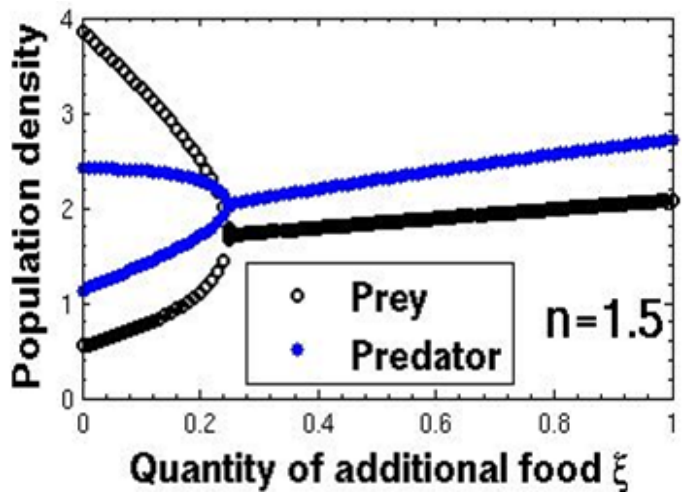




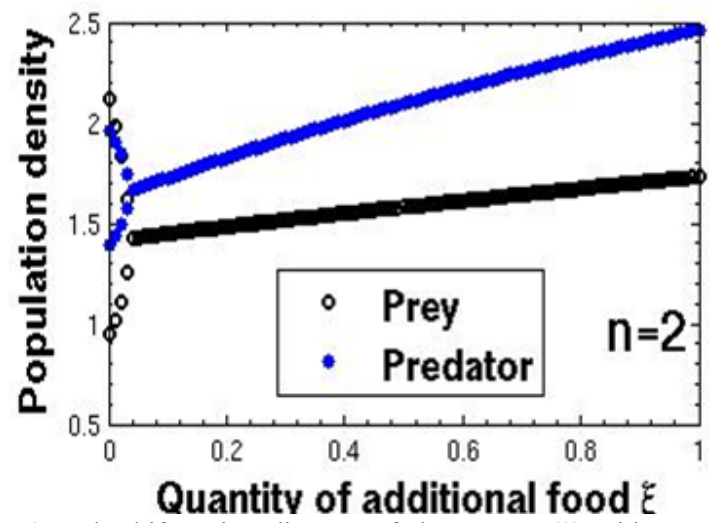

Figure 3. The bifurcation diagram of the system (3) with respect to quantity of additional food $\xi$ for different values of Holling parameter $n$

\section{Conclusions}

We have formulated a predator-prey model with general Holling interactions in presence of additional food to predator. We have discussed the dissipativeness of the system under certain conditions. The stability criteria of all equilibrium points of the system are derived. The numerical simulation is also shown here. We have seen that the dynamics of the system (3) highly depends on Holling parameter. There is extinction possibility for small values of Holling parameter without supply of additional food. Srinivasu et al. [8] defined that the supply of additional food should be high quality if $\alpha<1.5$ and low-quality if $\alpha>1.5$. Here, in figure 3 , we have shown that there is no extinction possibility in presence of high quality of additional food $(\alpha=1.1429, \xi=1.4)$ but for low-quality of additional food $(\alpha=2, \xi=1)$ the system has extinction risk for $0.2 \leq n<1.19$. Therefore, the existence of species in a system depends on interaction functions as well as quality of additional food. Here, we have shown that the model with Holling type-II or type-III is not always realistic. Because the model with Holling type-II or type-III is a particular case of general Holling interaction and this assumption is made for only simplicity of the model construction.
There is no reason for construction of such special type of interaction function. Therefore, we suggest that choice of interaction function is a very important part of realistic model for prediction of future determining biological strategy. Our study will be useful for construction of a real biological model and pest control.

\section{REFERENCES}

[1] A. Hastings T. Powell, 1991, Chaos in a three-species food chain, Ecology, 72, 896-903

[2] S. Gakkhar, A. Singh, 2012, Control of chaos due to additional predator in the Hastings-Powell food chain model, J Math. Anal. Appl., 385, 423-438

[3] Y. Do, H. Baek, Y. Lim and D. Lim, 2011, A three species food chain system with two types of functional responses. Abstract and Applied analysis, Article ID 934568, doi:10. $1155 / 2011 / 934569$

[4] T.V. Ton, A. Yagi, 2011, Dynamics of a stochastic predator-prey model with the Beddington-DeAngelis functional response, Communications on Stochastic Analysis, 2, 371-386

[5] D. Xiao, W. Li, M. Han, 2006, Dynamics in aratio- dependent predator-prey model with predator harvesting, J Math. Anal. Appl., 324, 14-29

[6] B. Mukhopadhyay, R. Bhattacharyya, 2011, On a three-tier ecological food chain model with deterministic and random harvesting: A mathematical study, Nonlinear Analysis: Modelling and Control, 16(1), 77-88

[7] T. Gross, W. Ebenhoh, U. Feudel, 2004, Enrichment and foodchain stability: the impact of different forms of predator-prey interaction, J. Theor. Biol., 227, 349-358

[8] P.D.N. Srinivasu, B.S.R.V. Prasad, M.Venkatesulu, 2007, Biological control through provision of additional food to predators:a theoretical study, Theor Popul Biol, 72, 111-120

[9] F. Cao and L. Chen, 1998, Asymptotic behavior of nonautonomous diffusive Lotka-Volterra model, System science and Mathematical Sciences, 11(2), 107-111 